\title{
The Expression of miR-31 and its Target Gene FOXP3 in Recurrent Implantation Failure Patients
}

\author{
Azita Azarpoor ${ }^{\circledR}$, Abdolreza Ardeshirylajimi $^{1}$, Samira Mohammadi Yeganeh $^{2,3}$, Elham Pour matrood $^{4}$, \\ Zeinab Dehghan ${ }^{5}$, Mohammad Salehi ${ }^{2,3^{*}}$
}

\begin{abstract}
Objectives: Endometrial receptivity is a complex event that occurs during the midluteal phase of the menstrual cycle known as the "window of implantation". During this period, the endometrium develops characteristics that allow the adhesion and invasion of the embryo to the uterine epithelium. Accordingly, the expressions of miR-31 and its target gene were evaluated to study the effect of miR-31 on FOPX3 gene expression in recurrent implantation failure (RIF) patients and normal fertile women. More precisely, the aim of this study was to understand the expression of miR-31 as one of the important regulators of the FOXP3 gene in the endometrium of RIF patients versus receptive endometria from fertile patients.

Materials and Methods: This case-control study was conducted on 20 endometrial tissue samples of normal fertile women and RIF patients in order to evaluate miR-31 and its target gene expression.

Results: According to the results of this study, a significant difference existed between RIF patients and normal fertile women (control group). The expression of the FOXP3 gene was more significant in the control group. miR-31 was also significantly expressed, which was due to the endometrial immunological disorder leading to the decreased expression of its target gene (FOXP3).

Conclusions: In general, implant abnormalities and recurrent abortions were observed in RIF patients due to the decreased expression of the FOXP3 gene resulting from the inhibitory effects of miR-31.

Keywords: microRNA, Endometrium, Recurrent implantation failure, FOXP3, miR-31
\end{abstract}

\section{Introduction}

MicroRNAs (mRNAs) are non-coding ribonucleic acids which are evolutionarily protected and regulate the gene expression after transcription by mRNA degradation or silencing or translational inhibition. In addition, as key regulators, mRNAs regulate a wide array of biological processes such as evolution, differentiation, proliferation, and apoptosis by controlling the expression of diverse genes $(1,2)$. Further, miRNAs make up only $0.01 \%$ of the total RNA (3). Scientists have estimated that there may be about 50,000 miRNAs in human cells which alter the function of thousands of genes and their protein synthesis. Furthermore, studies confirmed that miRNAs regulated about $30 \%$ of human genes (4). Pregnancy is an overcomplicated process that is influenced by multiple specific factors, each of which performs its function at a certain stage (i.e., fertilization, endometrial receptivity, and embryonic implantation). According to (5), endometrial receptivity is a prerequisite for establishing pregnancy which occurs in a restricted time range known as the "window of implantation" (WOI). The evaluation of the embryo implantation process indicated that the endometrial tissue plays a key role in this project. Uterine tissue by recruitment of immune cells (notably T-lymphocyte cells) and local immune tolerance mechanisms and also by secretion of cytokines, chemokines, growth factors, and adhesive molecules is prepared for embryo implantation. The slightest inconsistency in the expression or action of either of them can lead to implantation failure $(6,7)$. Repeated implantation failure (RIF) is the failure of the embryonic implantation into the uterus wall. It is estimated that approximately $10 \%$ of infertile patients have at least two or three RIFs after the in vitro fertilization (IVF) protocol (8). In human endometrium, several miRNAs have recently been identified which regulate the expression and function of several different genes (2,9). A panel of genes is effective in endometrial receptors, whose expression is controlled by a number of miRNAs such as miR 125b$5 p, 199 a-5 p$, and $31(9,10)$. Liu et al demonstrated the essential role of miR-30 in humans during the WOI of different menstrual cycle stages by the miRNAomic study technique. In addition, in the hamster, it was shown that the miR-30 family acts on the ubiquitin pathway by targeting ovarian proteins "Ubiquitin E3 ligase s-phase kinaseassociated protein 2" (2). In another study, Kresowik et 


\section{Key Messages}

miR-31 is an essential biomarker for endometrial receptivity that significantly elevated in the serum of fertile women.

- miR-31 has a direct effect on immunomodulatory factors such as FOXP3 that FOXP3 inhibits immune responses during embryo implantation.

- miR-31 overexpression caused the decreased expression of the FOXP3 gene leading to unstable endometrial receptivity and implantation failure.

al suggested that miR- 31 could be an essential biomarker for optimal endometrial receptivity. They proposed the theory that since the endometrium monthly disintegrates and collapses (9), the exosomes of endometrial secretions entering the circulatory system and thus the levels of the expression of peripheral blood miRNAs are measurable. In their study, miR-31 expression significantly elevated in the secretory phase in the serum of fertile individuals (9). Other experiments showed that miR-31 had a direct effect on immunomodulatory factors such as FOXP3 and CXCL12 expression levels (9). FOXP3 gene acts as an immune suppressor in endometrial receptors and inhibits immune responses during embryo implantation at the beginning of the secretory phase. Further, FOXP3 has generally been described as a master regulator of Treg development and function. Human FOXP3 has also been involved in nTreg function $(2,11)$. Considering the abovementioned explanations, this study aimed to develop previous studies by investigating the expression of the mir-31 gene and the subsequent expression of its target gene (FOXP3) in women with RIF and comparing them with normal fertile women (control group).

\section{Materials and Methods \\ Study Design}

This research was done at the Reproductive Medicine Unit of Payambaran Hospital, Tehran, Iran (2017-2019). A total of 20 women agreed to participate in our study. Ten endometrial specimens (RIF group) were collected from these women who were clinically diagnosed with RIF. Based on their diagnosis, they received an IVF cycle. In addition, 10 normal endometrial tissues were obtained from healthy women (control group).

Functional Classification and Protein-protein Interaction Network Analysis of FOXP3 Protein

Given that protein-protein interaction (PPI) networks are considered important for understanding the cellular processes and evolutionary properties of proteins (12), the present study used FOXP3 protein for constructing a PPI network in the STRING database (13). Next, the KEGG/BioCarta pathways of the network were assessed to identify the function of each highly connected region by the ClueGO app in Cytoscape software (14).
Primer Design for miRNA and its Target Gene

In reviewing the literature, the miR-31 was found, which is potentially overexpressed in RIF patients, and its target genes are determined by the specific software of miRWalk (http://www.miRwalk.umm.uni-heidelberg. de/), TargetScan (http://www.targetscan.org/), and Gene Runner, version 5.1 (Informer Technologies Inc., Spain). In addition, the primers were designed using Oligo 7 software (Molecular Biology Insights, Inc., Cascade, Company), and finally, validated with NCBI BLAST. The primer information is summarized in Table 1.

\section{Inclusion and Exclusion Criteria}

The inclusion criteria were being within the age range of 30-35 years and having the highest number of follicles (5-10 mm).

On the other hand, the exclusion criteria included tubal or male factors and unexplained infertility leading to IVF treatment, recurrent abortions attributed to hormonal, chromosomal, or anatomical causes, autoimmune diseases, metabolic or cardiovascular diseases, drinking and smoking histories, and ovarian hyperstimulation syndrome.

\section{Study Groups and Treatment}

In general, 20 individuals participated in this study, including 10 RIF patients having at least two abortions as the case group and 10 normal fertile women having at least all children of one sex and entering the IVF cycle for sex selection as the control group (Figure 1).

\section{In Vitro Fertilization}

According to the standard protocol, after ovulation induction in both groups, gonadotropin and antagonist injection (Aventis Pharma Company, Tokyo, Japan) was started since the third day of menstruation. In addition, the ultrasound-guided was done parallelly. Then, human chorionic gonadotropin (hCG, $10000 \mathrm{IU}$ ) was injected when the diameters of at least one of the follicles reached $20 \mathrm{~mm}$. Next, cytoplasmic sperm injection and follicular evacuation were done concurrently 36 hours after the hCG injection. Blastocysts were cultured for 48 to 72 hours and were finally frozen for future transfer.

Tissue Processing

Five days after the puncture, endometrial biopsy samples

Table 1. Designed Primers for qRT-PCR

\begin{tabular}{lll}
\hline Primer/Probe & Primer Sequence $\left(5^{\prime} \rightarrow \mathbf{3}^{\prime}\right)$ & Accession No. \\
\hline F FOXP3 & AAGGACAGCACCCTTTCGG & NM_014009.4 \\
R FOXP3 & GCTGCTCCAGAGACTGTAC & \\
F miR-31 & GTATACAGGCAAGATGCTGGC & NR_029505.1 \\
R miR-31 & GTGCAGGGTCCGAGGT & \\
\hline
\end{tabular}

Note. qRT-PCR: Quantitative real-time polymerase chain reaction. 


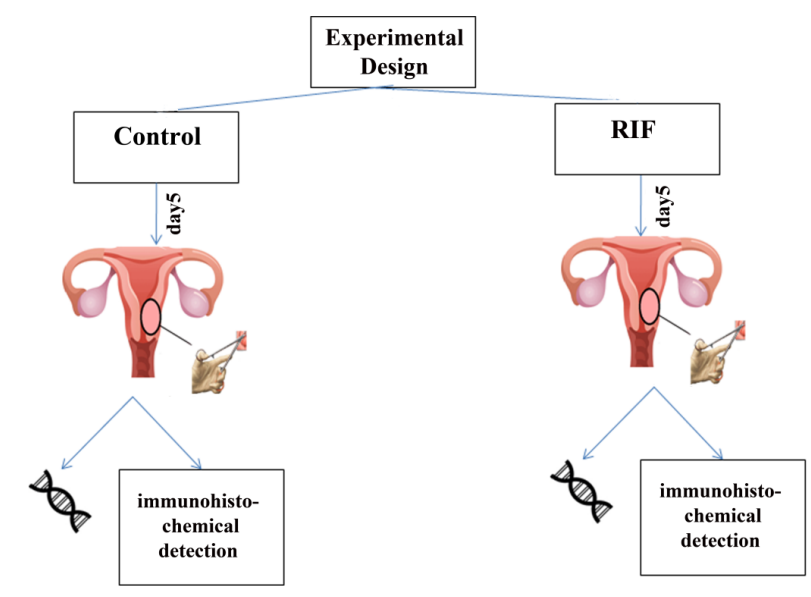

Figure 1. Schematic Diagram of Study Design.

were obtained using a pipelle sampler (Unimar, Inc., Wilton, CT, USA). The samples were divided into two sections. For the immunohistochemical studies of FOXP3 gene expression, one sample of the biopsy was maintained in $10 \%$ formalin while another part was stored in an RNAlater vial (Qiagen, Germany) and instantly frozen at $-80^{\circ} \mathrm{C}$ for molecular evaluations.

Messenger RNA (cDNA) Synthesis and RNA Extraction Total RNA and miRNA were extracted according to the protocol of kit isolation (GENE ALL, Korea). The RNA yield and its purity were examined on a nanodrop (Eppendorf, Germany), and single-strand cDNA was synthesized using YektaTajhiz kit (Tehran, Iran). Next, 2 $\mu \mathrm{L}$ random hexamer, $4 \mu \mathrm{L}$ RNA sample, $5 \mu \mathrm{L}$ nucleasefree water, $2 \mu \mathrm{L}$ miR 125b RT primer, $2 \mu \mathrm{L}$ miR 199a RT primer, and $2 \mu \mathrm{L}$ Snord47 RT primer were mixed in each microtube. Then, all samples were put on a thermocycler at $75^{\circ} \mathrm{C}$ for 5 minutes. Then, cDNA was synthesized using 5X RT buffer, $200 \mathrm{U}$ RT enzymes, $10 \mathrm{mmol} / \mathrm{L} \mathrm{dNTP}$, and a $10 \mathrm{U}$ RNase inhibitor. In the thermal cycler, reverse transcription reaction was carried out at $25,37,42,45$, and $72^{\circ} \mathrm{C}$ for $10,15,45$, and 10 minutes, respectively. Finally, the cDNA synthesis was performed by polymerase chain reaction (PCR) with GAPDH primers. The condition of PCR included $5 \mu \mathrm{L}$ master mix PCR (YektaTajhiz, Tehran, Iran), $0.5 \mu \mathrm{L}$ forward primer, $0.5 \mu \mathrm{L}$ reverse primer, $3 \mu \mathrm{L}$ water, and $1 \mu \mathrm{L}$ synthesized cDNAs. Then, all samples were put on a thermocycler at the primary denaturation, denaturation, annealing, extension, and the final extension at $95,95,60,72$, and $72^{\circ} \mathrm{C}$ for 5 minutes, 30 seconds, 45 seconds, 30 seconds, and 10 minutes, respectively, and eventually, loaded on a $2 \%$ agarose gel with a 50 bp ladder.

Quantitative Real-Time Polymerase Chain reaction According to the manufacturer's instructions of the standard kit of quantitative real-time polymerase chain reaction (qRT-PCR) (Gene All Biotechnology Company, South Korea), total RNA and microRNA were extracted for all specimens. The purity of extractions was assessed on a Nanodrop (Eppendorf, Germany) with an optical density (OD) of 260/280 nm, from which single-strand cDNA was synthesized using (YektaTajhiz kit, Tehran, Iran). Further, GAPDH gene primers were used to control and confirm cDNA synthesis using the PCR technique. Similarly, the Rotor-Gene Q instrument (Qiagen, Valencia, CA, USA) was used to evaluate the expressions of miR-31 and its target gene (FOXP3) by the qRT-PCR technique. The total volume of qRT-PCR was $13 \mathrm{~mL}$, including DNA Master SYBR Green I Mix (7 mmol/L, YektaTajhiz, Tehran, Iran), mixed forward and reverse primers $(1 \mathrm{mmol} / \mathrm{L})$, nucleasefree water $(4 \mathrm{mmol} / \mathrm{L})$ specific for miRNA and its target gene, and synthesized cDNA $(1 \mathrm{mmol} / \mathrm{L})$. The functional program of the qRT-PCR technique consisted of 40 cycles of extension for 2 minutes at $95^{\circ} \mathrm{C}$, denaturation for 5 seconds at $95^{\circ} \mathrm{C}$, annealing for 30 seconds at $60^{\circ} \mathrm{C}$, and 10 seconds at $72^{\circ} \mathrm{C}$ for amplification. The applied primers for RT-PCR are shown in Table 1.

\section{Histological Assessment}

Immunohistochemical analyses were carried out in deparaffinized and dehydrated samples in order to assess the FOXP3 protein from the endometrial tissues of the two groups. The recombinant anti-Foxp3 antibody (ab215206, Abcam) was used for the immunohistochemical distinction of FOXP3 at the dilutions of 1:100 concentration, and a $10 \%$ normal goat serum was utilized in phosphate buffer saline (PBS) for 20 minutes in order to block nonspecific binding. According to the working process of the protocol, the sections were incubated overnight with the antibody at $4^{\circ} \mathrm{C}$ and then were washed by PBS three times and incubated with the goat anti-rabbit immunoglobulin G (H\&L) antibody (FITC, ab6717, Abcam) for 1 hour anew. The sections were counterstained with DAPI, and the level of staining was assessed by OD evaluation using Image J software and the results were analyzed accordingly.

\section{Statistical Analysis}

Statistical analyses were done using SPSS version 16. RNA levels were analyzed by REST software and all diagrams were drawn by GraphPad Prism software. $P$ values $<0.05$ were considered statistically significant.

\section{Results}

Functional Classification and Protein-Protein Interaction Network

The results of the string showed that FOXP3 protein directly interacted with IL2RA, IL2, FOXP3, CTLA4, NFATC2, TNFRSF18, IFNG, RORC, RUNX1, GATA3, and IL10 proteins. Figure 2 depicts the entire network.

The results of the KEGG (Kyoto Encyclopedia of Genes and Genomes) pathway are presented in Table 2. The analysis of KEGG demonstrated that FOXP3 protein is an important regulator for Th17 cells and $\mathrm{T}$ cell receptors. 


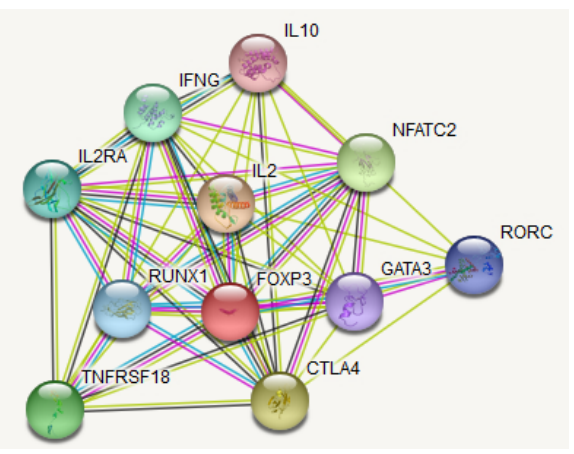

Figure 2. Protein-protein Interaction Network of FOXP3 and Associated Protein Constructed by String Database.

On the other hand, this protein has a key role in allograft rejection thus it can regulate implantation.

The Results of MiRNA and Target Gene Determination The findings of previous studies showed that our candidate miRNA was the miR-31, which originated from the 5'arm of its precursor. The miRwalk and Target scan algorithms predicted the putative targets of hsa-miR-31. FOXP3 is an important target gene which is found in the intersections of the two algorithms. One of its main targets, namely, FOXP3, was selected using the list of valid miR-31 targets provided through the miR base. FOXP3 is a transcription factor denoting $\mathrm{T}$ regulatory (Treg) compared in the window of implantation of both control and RIF patient's endometrium. Endometrial biopsy samples were obtained from 20 women whose clinical information is listed in Table 3 .

The Confirmation of cDNA Synthesis

The results of PCR with GAPDH primers (housekeeping gene) confirmed that cDNA synthesis was done correctly. Then, the detection showed a $145 \mathrm{bp}$ bond on the agarose gel (Figure 3).

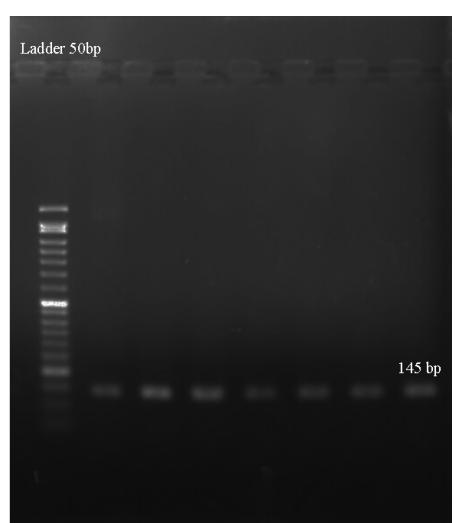

Figure 3. Controlling and Confirmation of cDNA Synthesis by GAPDH Gene Primers Using PCR. Note. PCR: Polymerase chain reaction.

Quantitative Measurement of the Expressions of miR-31 and its Target Gene FOXP3

According to the results of Figure 4, the qRT-PCR assessment of miR-31 represented significantly less (8fold) expression in the control group compared with the RIF group. Moreover, the expressions of FOXP3 (2.5fold) genes significantly increased in the control group compared to the RIF group (Figure 4).

Histological Assessment of Endometrial Tissue Samples The immunohistochemical detection of FOXP3 proteins was done and the results indicated that the control group had higher staining intensity of FOXP3 while the RIF group showed extremely weak staining for FOXP3 (Figure $5)$. Thus, the higher positive staining of FOXP3 protein is significant $(P<0.05)$ in the control group. To verify the histological results, the analysis revealed that the miR31 expression decreased in the control group, leading to an increase in FOXP3 gene expression and subsequently protein production of about $50 \%$ in the control group in comparison with the RIF group (Figure 6).

Table 2. KEGG Pathway Analysis for FOXP3 and Associated Proteins Performed by CluGO App in Cytoscape Software

\begin{tabular}{llll}
\hline GO* ID & GO* Term & $\boldsymbol{P}$ Value & Genes \\
\hline KEGG:04659 & Th17 cell differentiation & $2.07 \mathrm{E}-13$ & FOXP3, GATA3, IFNG, IL2, IL2RA, NFATC2, RORC, RUNX1 \\
KEGG:04660 & T cell receptor signaling pathway & $1.72 \mathrm{E}-07$ & CTLA4, IFNG, IL10, IL2, NFATC2 \\
KEGG:04658 & Th1 and Th2 cell differentiation & $1.08 \mathrm{E}-07$ & GATA3, IFNG, IL2, IL2RA, NFATC2 \\
KEGG:05330 & Allograft rejection & $1.92 \mathrm{E}-05$ & IFNG, IL10, IL2 \\
\hline
\end{tabular}

* Note. GO: gene ontology; KEGG: Kyoto Encyclopedia of Genes and Genomes.

Table 3. Clinical Information of Studied Groups

\begin{tabular}{llcccc}
\hline Studied Groups & Age & Infertility Duration & Number of Children & Number of Follicles (MII) & Number of Embryo (Good Quality) \\
\hline Control group & $30-35$ & - & $1-3$ & $8-11$ & $6-8$ \\
RIF group & $30-35$ & On average, 5-9 years & - & $7-10$ & $5-7$ \\
\hline
\end{tabular}

Note. RIF: Recurrent implantation failure. 


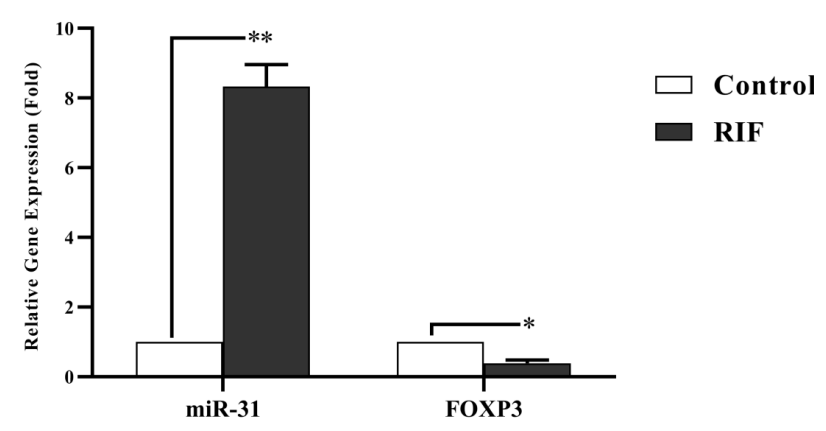

Figure 4. Expression Levels of microRNAs (miRNAs) and Genes in Endometrial Biopsy Samples
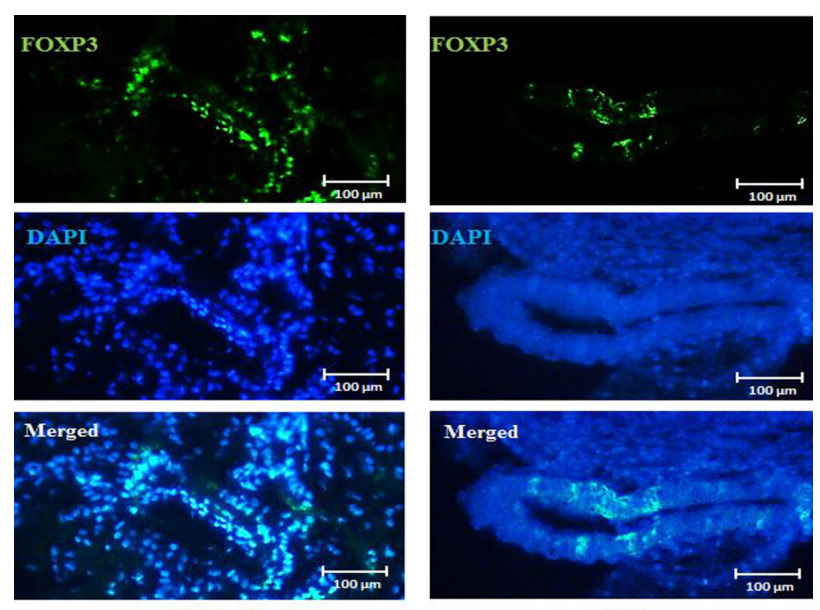

Control

RIF

Figure 5. Immunohistochemical Staining With DAPI for FOXP3 Gene and Comparing its Expression in the Two Studied Groups. Note. (A) Control group is identified as DAPI-positive (high expression) in the endometrial tissue. (B) RIF group is identified as low expression. Original magnification $\times 300$. *: A significant difference at $P<0.05$.

\section{Discussion}

Spontaneous abortion is highly common for the first time and given that it is not usually repeated, it does not need further investigations. However, couples and even the fetus and placenta should be examined after the second and third abortions in order to find its cause. Recurrent implantation failure or repeated implantation failure (RIF) refers to a situation that embryonic implantation has defected. The most important reasons for recurrent abortion are chromosomal abnormalities, uterine structural abnormalities, hormonal disorders, autoimmune diseases, and disorders associated with increased coagulation and infections (8). On the other hand, other factors such as specific drug consumption, exposure to environmental toxins, smoking, caffeine, placental disorders, father-related factors, and underlying maternal diseases (e.g., heart, blood, and kidney disease) are also effective in RIF. The immunological aspects of implantation failure are one of the most important attitudes in RIF evaluations. It has been suggested that the immune

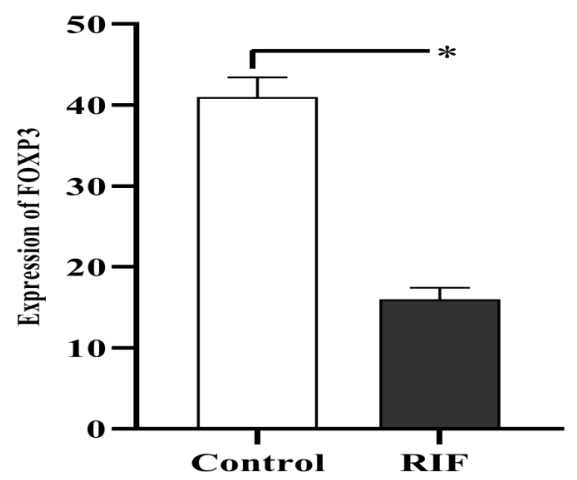

Figure 6. Downregulation of FOXP3 Protein in the RIF Group in Comparison to the Control Group. Note. RIF: repeated implantation failure.

cells as the regulators of endometrial acceptance may be used in RIF patients. Based on previous evidence, miR31 had a direct effect on immunomodulatory factors such as FOXP3 and CXCL12 expression levels (9). Recently, studies have focused on miRNAs and their functions in the endometrial acceptance and activity of target genes. For example, Fujita et al $(15,16)$ concluded that some immune cells can influence endometrial differentiation and the implantation window (WOI). Additionally, $\mathrm{Hu}$ et al showed that miR-21 with the RECK target gene in mice had high expressions at WOI thus suggested that miRNAs could play an important role in embryo implantation (17). In another study, Özcan et al described the role of the miR30 family in epithelial-to-mesenchymal transition $(18,19)$. In addition, $\mathrm{Ng}$ et al demonstrated the essential role of miR-30d in WOI. They reported that cellular exosomes containing specific miRNAs are present in uterine fluids and play a key role in embryo-endometrium cross-talk processes (20). Likewise, Revel et al identified 13 miRNAs, which differ in the endometrial receptivity in normal women than infertile individuals. Among these miRNAs, miR-99a, miR-23b, and miR-145 play an important role in endometrial dysfunction (21). Liu et al conducted many studies on the influence of miRNAs on endometrial function and concluded that mir-30 mediates endometrial secretions and fetal nutrition by secreting endometrial glands. They further showed that the direct target of miR101a and miR-199a in the rat is cyclooxygenase 2, which plays a key role in endometrial decidualization. They also concluded that increasing the miR-29a level leads to binding pro-apoptotic factors which inhibit cell apoptosis in the implantation process (2). The discovery of the FOXP3 gene as a transcriptional driver of Treg function demonstrated that the FOXP3 gene has been characterized in the production of $\mathrm{CD} 25+\mathrm{CD} 4+$ natural regulatory $\mathrm{T}$ cells which have a central role in the regulation of immune responses $(22,23)$. Based on the analysis of the PPI network, 10 important interactions were identified, which proved that the FOXP3 protein directly interacts with them. According to the finding of Table 3, an important pathway 
Table 3. Clinical Information of Studied Groups

\begin{tabular}{lccccc}
\hline Studied Groups & Age & Infertility Duration & No. of Children & Number of Follicles (MII) & Number of Embryo (Good Quality) \\
\hline Control group & $30-35$ & - & $1-3$ & $8-11$ & $6-8$ \\
RIF group & $30-35$ & On average, 5-9 years & - & $7-10$ & $5-7$ \\
\hline
\end{tabular}

Note. RIF: Recurrent implantation failure.

was found and its analysis (KEGG) showed that FOXP3 function leads to the regulation of Th17 cells and T cell receptors which have a key role in regulated implantation and confirms the results of other studies. Overall, this study evaluated miRNA (mir-31) and its target gene (FOXP3) expression patterns in normal fertile endometria at WOI and then compared the findings with those of RIF patients. Although previous studies investigated miRNAs in connection with endometrial receptivity, no study has so far focused on the simultaneous evaluation of mir-31 and its target gene (FOXP3) in patients with recurrent miscarriage compared to healthy women. The results of our study showed a 2.5-fold increase in FOXP3 gene expression in healthy women compared to RIF patients, and mir-31 expression in RIF patients represented an 8 -fold increase compared to healthy women. Thus, the results revealed a significant difference between RIF patients (RIF group) and normal fertile women (control group). The expressions of the FOXP3 gene were highly significant in comparison with the control group, which is consistent with the results of other previously mentioned studies. miR-31 was also significantly expressed, which was due to the endometrial immunological disorder leading to the decreased expression of its target gene (FOXP3). Furthermore, implant abnormalities and recurrent abortions were observed in RIF patients due to the decreased expression of the FOXP3 gene that results from the inhibitory effects of mir-31.

\section{Conclusions}

To the best of our knowledge, this is the first and a novel study that identified the role of miR-31 in FOXP3 gene expressions. We expected a decrease in FOXP3 gene expressions in RIF patients while an increase in their regulatory microRNAs and normal fertile women (control group) although the opposite happened that provided a suitable endometrial environment for embryo development and attachment. The results of this study showed that miR-31 expression caused the decreased expression of the FOXP3 gene leading to unstable endometrial receptivity and implantation failure.

\section{Authors' Contributions}

The project was conducted and written by AzA. In addition, MS was the team leader and supervised the project and $\mathrm{AbA}$, as the second supervisor, edited the manuscript. Further, EP and $\mathrm{ZH}$ revised the manuscript and provided additional revisions, support, and guidance in collaboration with SMY and ZD. All authors contributed to manuscript revision, reading, and approving the submitted version.

\section{Conflict of Interests}

Authors declare that they have no conflict of interests.

\section{Ethical Issues}

The Ethics Committee of Shahid Beheshti University of Medical Sciences approved this study (Approval No. IR.SBMU.RETECH.REC.1396.999). All experimental conditions were explained to participants, and they were required to legally and ethically sign an informed consent form for participating in this trial.

\section{Financial Support}

The authors would like to thank the Cellular and Molecular Biology Research Center, Shahid Beheshti University of Medical Sciences for funding this project.

\section{Acknowledgments}

This article was extracted from a Ph.D. thesis submitted by Azita Azarpoor. We express our appreciation to the Cellular and Molecular Biology Research Center at Shahid Beheshti University of Medical Sciences and Payambaran Hospital, Tehran, Iran.

\section{References}

1. Vallian Broojeni S, Kheradmand P. Bioligy, function and detection of microRNA. Laboratory \& Diagnosis. 2015;7(28):33-40. [Persian].

2. Liu W, Niu Z, Li Q, Pang RT, Chiu PC, Yeung WS. MicroRNA and embryo implantation. Am J Reprod Immunol. 2016;75(3):263-271. doi:10.1111/aji.12470

3. Fakhr E, Motamed N, Habibi Rezaei M. Microarray technology. Genetics in The 3rd Millennium. 2011;9(3):2481-2488. [Persian].

4. Mukherji S, Ebert MS, Zheng GX, Tsang JS, Sharp PA, van Oudenaarden A. MicroRNAs can generate thresholds in target gene expression. Nat Genet. 2011;43(9):854-859. doi:10.1038/ng.905

5. Altmäe S, Martinez-Conejero JA, Esteban FJ, et al. MicroRNAs miR-30b, miR-30d, and miR-494 regulate human endometrial receptivity. Reprod Sci. 2013;20(3):308317. doi:10.1177/1933719112453507

6. Dimitriadis E, White CA, Jones RL, Salamonsen LA. Cytokines, chemokines and growth factors in endometrium related to implantation. Hum Reprod Update. 2005;11(6):613-630. doi:10.1093/humupd/dmi023

7. Rahimipour M, Salehnia M, Jafarabadi M. Alteration of genes expression related to implantation in human 
endometrial stromal cells cultured in the presence of ovarian hormones. Pathobiology Research. 2017;20(1):2942. [Persian].

8. Coughlan C, Ledger W, Wang Q, et al. Recurrent implantation failure: definition and management. Reprod Biomed Online. 2014;28(1):14-38. doi:10.1016/j. rbmo.2013.08.011

9. Kresowik JD, Devor EJ, Van Voorhis BJ, Leslie KK. MicroRNA-31 is significantly elevated in both human endometrium and serum during the window of implantation: a potential biomarker for optimum receptivity. Biol Reprod. 2014;91(1):17. doi:10.1095/biolreprod.113.116590

10. Lin X, Beckers E, Mc Cafferty S, et al. Bovine embryosecreted microRNA-30c is a potential non-invasive biomarker for hampered preimplantation developmental competence. Front Genet. 2019;10:315. doi:10.3389/ fgene.2019.00315

11. Kim CH. FOXP3 and its role in the immune systemAdv Exp Med Biol. 2009;665:17-29. doi:10.1007/978-1-44191599-3_2

12. Schwartz AS, Yu J, Gardenour KR, Finley RL Jr, Ideker T. Cost-effective strategies for completing the interactome. Nat Methods. 2009;6(1):55-61. doi:10.1038/nmeth.1283

13. Szklarczyk D, Gable AL, Lyon D, et al. STRING v11: protein-protein association networks with increased coverage, supporting functional discovery in genomewide experimental datasets. Nucleic Acids Res. 2019;47(D1):D607-D613. doi:10.1093/nar/gky1131

14. Bindea G, Mlecnik B, Hackl H, et al. ClueGO: a Cytoscape plug-in to decipher functionally grouped gene ontology and pathway annotation networks. Bioinformatics. 2009;25(8):1091-1093. doi:10.1093/bioinformatics/btp101

15. Virant-Klun I, Ståhlberg A, Kubista M, Skutella T. MicroRNAs: from female fertility, germ cells, and stem cells to cancer in humans. Stem Cells Int. 2016;2016:3984937. doi:10.1155/2016/3984937

16. Fujita K, Nakayama T, Takabatake K, et al. Administration of thymocytes derived from non-pregnant mice induces an endometrial receptive stage and leukaemia inhibitory factor expression in the uterus. Hum Reprod. 1998;13(1o):28882894. doi:10.1093/humrep/13.10.2888

17. Zhang Y, Wang Q, Wang $\mathrm{H}$, Duan E. Uterine fluid in pregnancy: a biological and clinical outlook. Trends Mol Med. 2017;23(7):604-614. doi:10.1016/j. molmed.2017.05.002

18. Ruiz-Alonso M, Blesa D, Díaz-Gimeno $\mathrm{P}$, et al. The endometrial receptivity array for diagnosis and personalized embryo transfer as a treatment for patients with repeated implantation failure. Fertil Steril. 2013;100(3):818-824. doi:10.1016/j.fertnstert.2013.05.004

19. Ozcan S. MiR-30 family and EMT in human fetal pancreatic islets. Islets. 2009;1(3):283-285. doi:10.4161/isl.1.3.9968

20. Ng YH, Rome S, Jalabert A, et al. Endometrial exosomes/ microvesicles in the uterine microenvironment: a new paradigm for embryo-endometrial cross talk at implantation. PLoS One. 2013;8(3):e58502. doi:10.1371/ journal.pone.0058502

21. Revel A, Achache H, Stevens J, Smith Y, Reich R. MicroRNAs are associated with human embryo implantation defects. Hum Reprod. 2011;26(10):2830-2840. doi:10.1093/ humrep/der255

22. Ofoghi M, Mesdaghi M, Khalilnezhad A, Taheri-Panah R, Amani D. Evaluation of CD4+CD25+FOXP3+ regulatory $\mathrm{T}$ cells in tumor environment of invasive intraductal breast carcinoma and control group. Research in Medicine. 2015;39(2):55-59. [Persian].

23. Tang Q, Bluestone JA, Kang SM. CD4(+)Foxp3(+) regulatory $\mathrm{T}$ cell therapy in transplantation. J Mol Cell Biol. 2012;4(1):11-21. doi:10.1093/jmcb/mjr047

(c) 2020 The Author(s); This is an open-access article distributed under the terms of the Creative Commons Attribution License (http:// creativecommons.org/licenses/by/4.0), which permits unrestricted use, distribution, and reproduction in any medium, provided the original work is properly cited. 